\title{
CLINICAL, CYTOLOGICAL AND HISTOPATHOLOGICAL CORRELATION IN LESIONS OF UTERINE CERVIX
}

\author{
Jita Parija', Janmejaya Mohapatra ${ }^{2}$
}

${ }^{1}$ Associate Professor, Department of Gynaecological Oncology, Acharya Harihar Regional Cancer Centre, Cuttack, Odisha. ${ }_{2}^{2}$ Assistant Professor, Department of Gynaecological Oncology, Acharya Harihar Regional Cancer Centre, Cuttack, Odisha.

ABSTRACT
BACKGROUND
Cytological screening of the uterine cervix by Papanicolaou test has reduced the incidence of cervical cancer. But cytological
diagnosis in clinically suspicious lesions of cervix is provisional and has to be corroborated with histopathological findings for
effective treatment response.

\section{MATERIALS AND METHODS}

Study Design- A descriptive study.

Three hundred patients attending Gynaecological Oncology OPD of A.H. Regional Cancer Centre, Cuttack during the period April 2016-March 2017, were studied. History taking, clinical examination was followed by cervical smear and multiple punch biopsies, in patients with unhealthy cervix. The cytological and histopathological results were correlated with each other and clinically.

\section{RESULTS}

Clinically, cancer was detected in $77.6 \%$ cases. Cytologically, $73.3 \%$ reported carcinoma and 3.33\% squamous intraepithelial lesions. Histopathologically, $74.6 \%$ showed carcinoma and $3.7 \%$ SIL. The cytohistopathological agreement was $69.2 \%$ in inflammatory lesions and $90.5 \%$ in invasive carcinomas. In clinically unhealthy cervix, a report of squamous intraepithelial-lesion by cytology was seen in $17.02 \%$ and by histopathology was found in $19.15 \%$. Cytological proof of cancer was obtained in $73.3 \%$ of cases and histopathological proof in 74\%. On overall analysis of 300 cases of suspicious cervix, 229 correlated well with cytology, labelling them as abnormal and 246 cases were stamped as diseased by histopathology.

\section{CONCLUSION}

This study proves that cytology can be used as a very effective screening tool to catch high risk cases, which can be biopsied to detect cancer early. Both cytology and histopathology correlate well with clinically obvious lesions of the cervix.

\section{KEYWORDS}

Cytology, Histopathology, Correlation, Lesion, Cervix.

HOW TO CITE THIS ARTICLE: Parija J, Mohapatra J. Clinical, cytological and histopathological correlation in lesions of uterine cervix. J. Evolution Med. Dent. Sci. 2017;6(64):4652-4655, DOI: 10.14260/Jemds/2017/1006

\begin{abstract}
BACKGROUND
Cancer of uterine cervix is the second most common cancer worldwide next to breast cancer and most common female cancer in many developing countries like India. Cervical cancer is an important cause of morbidity and mortality among females worldwide. With the effective implementation of screening procedure, awareness program, education, improved quality of living; incidence of carcinoma cervix has drastically decreased in developed countries; however, it is still one of the common neoplasm in developing countries. In India, it is one of the most common neoplasms in females with an incidence of $14.42 / 1,00,000$ population and mortality rate of $2.83 / 1,00,000$ population. The age adjusted incidence rates (AARs) of cervical cancer has decreased in the urban Indian population.
\end{abstract}

Financial or Other, Competing Interest: None.

Submission 15-06-2017, Peer Review 29-07-2017,

Acceptance 04-08-2017, Published 10-08-2017.

Corresponding Author:

Dr. Jita Parija,

Parijas, Mahatab Road,

Cuttack-753012, Odisha.

E-mail:jita049@gmail.com

DOI: $10.14260 /$ jemds/2017/1006
However, over 70 percent of the Indian population resides in the rural areas. Cancer of the cervix still constitutes the number one cancer in India. WHO global report 2014 states 266000 women died from cervical cancer in year 2012 .

The cervical epithelium presents a spectrum of cervical intraepithelial neoplasia (CIN) changes as precancerous state. Most cervical cancers can be detected at a pre-invasive state with an adequate cytological screening and treated appropriately thus preventing overt progression of the lesion to full blown cancer and in turn decreasing morbidity and mortality.

With the effectiveness of the Papanicolaou (PAP) cytologic test in early detection of precancerous state and accessibility of cervical biopsy the mortality rate due to cervical carcinoma has declined statistically.

PAP smear screening done every two years starting at the age of 21 years offers the best chance of preventing cervical cancer. The use of the cervical smear (Papanicolaou) as a screening tool has significantly reduced the incidence of cervical cancer. Cervical smears include cells exfoliated from body of the uterus. (Even at times from fallopian tubes and ovaries). Cervical biopsy is usually done after an abnormality has been found during a routine pelvic exam or Pap smear. Cytohistopathological correlation of Pap smear is a widely accepted method of internal quality assurance and helps in the analysis of various factors leading to discrepant diagnosis. With the above view, the present study has been carried out 
to evaluate the cytohistopathological correlation of the various cervical lesions. The concept of pre-invasive disease of the cervix was first introduced by Papanicolaou in 1947 and since then, cervical cytological testing has become the standard screening test for cervical neoplasm. Cervical cytological screening is designed to detect over $90 \%$ of cytological abnormalities. It has also been seen that most cervical cancers can be diagnosed at the pre-invasive stage with adequate and repetitive cervical cytological screening. Cytological examination by Papanicolaou smears is not only confined to the diagnosis of premalignant and malignant lesions of the uterine cervix but also for identifying the reactive and infective conditions. Cervical smears may include cells exfoliated from body of the uterus and even at times from fallopian tubes and ovaries.

The potential threat of cancer is the main reason for Pap smear screening programs and if abnormalities are detected, they have to be confirmed subsequently by histopathologic examination. Pathologists are frequently faced with the problem of reconciling the cytology report with the histopathological findings. Most of the cases of cervical cancer are caused by infection with oncogenic or high-risk types of Human Papilloma Virus. HPV-16 and HPV-18 are the two types that cause most of the cervical cancers. Human papilloma virus (HPV) is recognised as the primary causal factor in the development of cervical cancer. The accessibility of the uterine cervix with propensity of its cells to exfoliate and a prolonged natural history, makes Pap smear feasible. Pap smear as a screening tool has significantly reduced the incidence of cervical cancer. Cytohistopathological correlation of Pap smear is a widely accepted method of internal quality assurance and helps in the analysis of various factors leading to discrepant diagnosis. With the above view, the present study has been carried out to evaluate the cytohistopathological correlation of the various cervical lesions.

The success of screening for cervical cancer is based on collection of adequate materials and correct interpretation of abnormal cells.

History of premalignant lesions provides the best potential for the control of cancer by population screening. Although Papanicolaou (Pap) cytology represents the most effective technique to prevent and detect the precancerous conditions of the uterine cervix before they become invasive cancer, its false negative yield due to the potential sampling and interpretation errors yield is still a reason of concern. Thus, the final diagnosis should be made on histologic examination to assess the accuracy of cervical cytology. Cytohistopathological correlation of Pap smear is one of the recommendations of the European guidelines for quality assurance for the development of cytology laboratory performance and, in particular, to reduce false negative results.

Routine cytological screening of the uterine cervix based on Papanicolaou test, has reportedly reduced the incidence of invasive carcinoma cervix ${ }^{1 \& 2}$ (Walton RJ 1976, Macgregor 1982). Cytological diagnosis in clinically suspicious lesion is to be fortified with biopsy and colposcopically directed biopsy, if required. Histopathological grading in invasive carcinoma of cervix, carries importance in the nature and behaviour of the disease and its response to treatment. A difference in cytological and histopathological opinion is often encountered and preference in final diagnosis is given to histopathological report. However, both should be taken as reciprocative to each other and ought to be correlated clinically with the lesion. In the present descriptive study, effort has been made to analyse the clinical picture of the cervix with its corresponding cytological and histopathological diagnosis.

\section{MATERIALS AND METHODS}

Consecutive three hundred cases attending the Gynaecological Oncology OPD of A. H. Regional Cancer Centre, Cuttack over the period April 2016 to May 2017, were taken up for a retrospective analysis. All cases were subjected to history taking and clinical examination. Patients with unhealthy cervix were subjected to cervical smear and multiple punch biopsy.

Cervical smears were obtained by Gynaecologists, using Ayres spatula. Smears were covered with Biofix spray, and sent to Pathology department. These smears were immersed in Papanicolaou fixative (equal volumes of $95 \%$ ethanol and ether) for minimum of 30 minutes and then stained with Papanicolaou stain.

Cytological features in these smears were examined in detail. Histopathological specimens were grossed, processed and blocks were made. Then sections of 3-5 microns were prepared, stained with H\&E stain and microscopically examined. Adequacy of the cytological material: Satisfactory smears which consisted of well preserved and well visualised squamous cells, that are spread over more than $10 \%$ of the slide surface, and with adequate endocervical/transformation zone component, (minimum of two clusters of well-preserved endocervical and/or squamous metaplastic squamous cells), were only included in the study.

Biopsy specimens were fixed in $10 \%$ formalin, and were processed by routine procedure to obtain paraffin sections, and were stained with Haematoxylin and Eosin.

Histopathology reporting was done separately without the knowledge of cytology report. Later cytological features were correlated with histopathological findings. The results were correlated with each other.

\section{RESULTS}

\begin{tabular}{|c|c|}
\hline Age in Years & \% of Cases \\
\hline $20-29$ & $2.4 \%$ \\
\hline $30-39$ & $14.93 \%$ \\
\hline $40-49$ & $37.15 \%$ \\
\hline $50-59$ & $29.86 \%$ \\
\hline $60-69$ & $15.97 \%$ \\
\hline \multicolumn{2}{|c|}{ Table 1. Age - Distribution } \\
\hline
\end{tabular}

Age range of the study group was 20-70 years and majority were in the $40-49$ years age group.

\begin{tabular}{|c|c|c|}
\hline Clinical Appearance & No. of Cases & \% \\
\hline Unhealthy & 47 & $15.6 \%$ \\
\hline Cervicitis & 13 & $4.33 \%$ \\
\hline Erosion & 07 & $2.33 \%$ \\
\hline Growth/Ulcer & 233 & $77.7 \%$ \\
\hline \multicolumn{2}{|c|}{ Table 2. Clinical Appearance } \\
\hline \multicolumn{2}{|c|}{}
\end{tabular}


Clinically, $77.7 \%$ presented with overt growth, $15.6 \%$ as unhealthy cervix and $2.33 \%$ and $4.33 \%$ as erosion and cervicitis, respectively.

\begin{tabular}{|c|c|c|}
\hline Cytology & No. of Cases & \% \\
\hline Normal & 35 & $11.7 \%$ \\
\hline Inflammatory & 39 & $13 \%$ \\
\hline LSIL & 01 & $0.33 \%$ \\
\hline HSIL & 09 & $3 \%$ \\
\hline Invasive Cancer & 220 & $73.3 \%$ \\
\hline \multicolumn{2}{|c|}{ Table 3. Distribution According to Cytology } \\
\hline
\end{tabular}

High grade intraepithelial lesion was reported in 3\% and invasive cancer in $73.3 \%$ of cases by cytology.

\begin{tabular}{|c|c|c|}
\hline HP & No. of Cases & $\mathbf{\%}$ \\
\hline Normal & 02 & $0.7 \%$ \\
\hline Inflammatory & 65 & $21.6 \%$ \\
\hline LSIL & 02 & $0.7 \%$ \\
\hline HSIL & 09 & $3 \%$ \\
\hline Invasive Cancer & 222 & $74 \%$ \\
\hline \multicolumn{2}{|c|}{ Table 4. Distribution According } \\
According to Histopathology \\
\hline
\end{tabular}

HSIL was reported in $3 \%$ and invasive cancer in $74 \%$ of cases by histopathological studies.

\begin{tabular}{|c|c|c|c|}
\hline Diagnosis & Cytology-No. & Biopsy-No. & $\mathbf{\%}$ \\
\hline Inflammatory & 39 & 65 & $69.2 \%$ \\
\hline LSIL & 01 & 02 & $50 \%$ \\
\hline HSIL & 09 & 09 & $100 \%$ \\
\hline Invasive Cancer & 220 & 222 & $90.5 \%$ \\
\hline
\end{tabular}

The agreement between cytology and histopathology was $69 \%$ in inflammatory lesions. Maximum correlation was seen in HSIL and invasive cancer.

\begin{tabular}{|c|c|c|c|}
\hline Diagnosis & $\begin{array}{c}\text { Clinical } \\
\text { Cases }\end{array}$ & $\begin{array}{c}\text { Cytological } \\
\text { Agreement }\end{array}$ & $\begin{array}{c}\text { HP } \\
\text { Agreement }\end{array}$ \\
\hline Inflam/Erosion & 20 & $95 \%$ & $100 \%$ \\
\hline Unhealthy Cx/SIL & 47 & $17.02 \%$ & $19.5 \%$ \\
\hline Inv-Ca & 233 & $86.7 \%$ & $93.13 \%$ \\
\hline Total & $\mathbf{3 0 0}$ & $\mathbf{7 6 . 3 \%}$ & $\mathbf{8 2 \%}$ \\
\hline \multicolumn{3}{|c|}{ Table 6. Agreement between Clinical } \\
Picture and Cytopathological Results \\
\hline
\end{tabular}

Clinico-cytohistopathological agreement, was best achieved in inflammatory and carcinomatous lesions of cervix. Statistical agreement between cytology and histopathology in different lesions of cervix was done and the results are given below.

\section{Inflammation}

\begin{tabular}{|c|c|c|c|}
\hline Inflammation & +ve & -ve & Marginal Row Totals \\
\hline Cytological findings & 19 & 1 & 20 \\
\hline Histopathology & 19 & 1 & 20 \\
\hline $\begin{array}{c}\text { Marginal Column } \\
\text { Totals }\end{array}$ & 38 & 2 & 40 (Grand Total) \\
\hline
\end{tabular}

The chi square statistic is 0 . The $p$ value is 1 . This result is not significant at $\mathrm{p}<0.05$.

The high value of $p$ indicated that the findings of Cytology and Histopathology are not different.

\section{Unhealthy $\mathrm{Cx} / \mathrm{SIL}$}

\begin{tabular}{|c|c|c|c|}
\hline Test & +ve & -ve & Marginal Row Totals \\
\hline Cytology & 8 & 39 & 47 \\
\hline Histopathology & 9 & 38 & 47 \\
\hline $\begin{array}{c}\text { Marginal Column } \\
\text { Totals }\end{array}$ & 17 & 77 & 94 (Grand Total) \\
\hline
\end{tabular}

The chi square statistic is 0.0718 . The $p$ value is 0.788719 . This result is not significant at $\mathrm{p}<0.05$.

The high value of $p$ indicated that the findings of Cytology and Histopathology are not different.

\section{Invasive Carcinoma}

\begin{tabular}{|c|c|c|c|}
\hline Test & +ve & -ve & Marginal Row Totals \\
\hline Cytological findings & 202 & 31 & 233 \\
\hline Histopathology & 217 & 16 & 233 \\
\hline $\begin{array}{c}\text { Marginal Column } \\
\text { Totals }\end{array}$ & 419 & 47 & $\begin{array}{c}466 \\
\text { (Grand Total) }\end{array}$ \\
\hline
\end{tabular}

The chi square statistic is 5.3242 . The p value is 0.021031 . This result is significant at $p<0.05$. Two findings are significantly different.

\section{DISCUSSION}

Prevalence of carcinoma cervix worldwide is 3,95,5000/year and mortality is $1,95,000 /$ year. Women under 45 years of age have $2 \%$ chance of developing cervical cancer. However, screening programs have reduced the incidence and mortality from carcinoma cervix in developed countries. It is well accepted that PAP smear has been the most effective cancer screening test ever introduced. The screening test has achieved a reduction in the death rate of $>70 \%$ for a prevalent cancer.

The success of PAP test has resulted in unrealistic community expectations, with a consequent rise in litigation. It is important to be aware that as with any pathological tests, there are recognisable false results for PAP test. Traditionally, the gold standard for assessing the performance of PAP smears prediction has been the histology of cervical biopsies. Although it is acknowledged that histology also suffers certain drawbacks, since it is to a lesser extent, it nevertheless, provides a reasonable parameter to gauge the performance of the Pap test. The incidence of cervical carcinoma is incredibly high. Almost $80 \%$ of all its cases are attributed to developing countries, because of lack of proper knowledge, long intervals between tests and non-existent prevention programmes. It is known that Pap smear has been the most effective cancer screening test ever introduced. This screening test has helped achieve a reduction in the death rate of more than $70 \%$ for a prevalent cancer. Carcinoma cervix is thought to be an ideal gynaecological malignancy for screening as it fulfils both test and disease criteria for screening ${ }^{1}$ (Walton RJ). Because of its long latent phase, it can be detected as identifiable and treatable premalignant lesion which precedes the invasive disease. 
Screening by Pap Smear is more than worth its cost ${ }^{2}$ (Macgregor JE, 1982). The original terminology of dysplasia and carcinoma in situ (WHO) classification was replaced by the cervical intraepithelial neoplasia (CIN) terminology. Recently the Bethesda system of terminology has been introduced to sub-classify the lesions into grades: high grade and low grade Squamous Intraepithelial Lesions (SIL). Biopsy report is considered as the gold standard provided biopsy is taken from the appropriate site. Pap smear test is inexpensive, non-invasive and closely as sensitive, specific and effective as biopsy. However, biopsy can be performed if any abnormalities are detected in Pap smear.

Since A.H. Regional Cancer Centre Cuttack is a referral hospital, the three hundred consecutive cases studied, were mainly patients with suspicious cervix, referred for confirmation of the diagnosis and further treatment. Liquid based pap cytology test was not performed. In this study, squamous-intraepithelial lesion was observed in 3.33\% and invasive cancer in $73.3 \%$ respectively ${ }^{3,4}$ (MB Tengli, 2016 \& Edwardo L. Franco et al, 2001) which is different from studies conducted in other non-gynaecological centres. In contrast, a population-based study by Meisels and Morin $^{5}$ (1985), revealed squamous intraepithelial lesion in $0.32 \%$ and invasive cancer in $0.05 \%$ of cases only. In referral centres catering to treatment of carcinoma, this discrepancy is quite clearly expected. The disparity in results of cytology and histopathology (Table V), comes to an overall figure of $36.85 \%$; which is otherwise called the false negative rate 6 (Vaishali et al, 2010). This is higher than that of the overall false negative rate $12 \%$, response by Lambourne ${ }^{7} \mathrm{~A}$ and Lederer $\mathrm{H}$ et al, 1973. The false negative rate of $9.47 \%$ for invasive carcinoma, however, corresponds well with that figure. The false negative results in histopathology and also cytology is due to necrosis, inflammation, inadequate smear collection and biopsies from unaffected areas. Discrepancies in cytological and histopathological diagnoses may be attributed to either inadequate sampling, or errors in biopsy techniques. Therefore, in clinically suspicious lesions, repeat smears and repeat biopsies are strongly recommended, before deciding for the line of treatment.

\section{CONCLUSION}

PAP smear test is found to be a sensitive and highly effective method for the detection of cervical neoplastic changes. Cytological features significantly correlate with histological findings. Thus, screening procedures should be implemented in early age in sexually active females to detect the lesion in an early stage. Pap smear can be used to detect most common problem of infection in cervical region. Screening for cancer is an important aspect. PAP smear is a less invasive and simple procedure to perform. It is cheap and affordable to detect early onset abnormalities on regular basis. Cervical carcinoma does not develop suddenly from normal epithelium. It is presented by a spectrum of intraepithelial neoplastic changes which are precancerous lesions and were termed as cervical intraepithelial lesions. Pap smear test was found to be equally sensitive to histopathological examination for the early detection of different cervical lesions. The effective role of exfoliative cytology in detecting the cellular abnormalities of the cervix is beyond doubt. However, it is advised to perform biopsy if any abnormalities are detected in Pap smear for correlation and confirmation. Pap smear screening should still continue according to past guidelines to minimise cancer incidence and all cases of squamous intraepithelial lesions should be subjected to histopathological study to rule out carcinomas.

\section{REFERENCES}

[1] Walton RJ. Cervical cancer screening programs. V. Conclusions and recommendations of the task force. Can Med Assoc J 1976;114(11):1003.

[2] MacGregor JE. Screening for preclinical cervical cancer. J College of Obstetricians and Gynaecologists 1982:95-107.

[3] Tengli MB. A cyto-histopathological correlation study of lesions of uterine cervix. oaji,net/pdf.html?n = 2016/1770-146760708pdf.

[4] Franco EL, Duarte-Franco E, Ferenczy A. Clinico pathological study of erosion of cervix. CMAJ 2001;164(7):1017-25.

[5] Meisels A, Morin C. Human papillomavirus and cancer of the human cervix. Gynecol Oncol 1981;12(2 Pt 2):S111-23.

[6] Vaishali J, Vyas AS. Cervical neoplasia-cyto-histological correlation (Bethesda system)-a study of 276 cases. Journal of Cytology and Histology-Open Access 2010;1(2):1000106.

[7] Lambourne A, Lederer H. Effects of observer variation in population screening for cervical carcinoma. J Clinical Pathology 1973;26(8):564-9. 